\title{
Direct Index Method of Beam Damage Location Detection Based on Difference Theory of Strain Modal Shapes and the Genetic Algorithms Application
}

\author{
Bao Zhenming, ${ }^{1}$ Zhao Deyou, ${ }^{1}$ Lin Zhe, ${ }^{1}$ \\ Ma Jun, ${ }^{1}$ and Zhen Yaobao ${ }^{2}$ \\ ${ }^{1}$ Department of Naval Architecture and Ocean Engineering, Dalian University of Technology, \\ Dalian 116024, China \\ ${ }^{2}$ School of Mechanical Engineering, Dalian University of Technology, Dalian 116024, China \\ Correspondence should be addressed to Zhen Yaobao,xu_xia@yahoo.cn
}

Received 5 October 2012; Accepted 14 November 2012

Academic Editor: Rui Mu

Copyright (C) 2012 Bao Zhenming et al. This is an open access article distributed under the Creative Commons Attribution License, which permits unrestricted use, distribution, and reproduction in any medium, provided the original work is properly cited.

\begin{abstract}
Structural damage identification is to determine the structure health status and analyze the test results. The three key problems to be solved are as follows: the existence of damage in structure, to detect the damage location, and to confirm the damage degree or damage form. Damage generally changes the structure physical properties (i.e., stiffness, mass, and damping) corresponding with the modal characteristics of the structure (i.e., natural frequencies, modal shapes, and modal damping). The research results show that strain mode can be more sensitive and effective for local damage. The direct index method of damage location detection $I_{\mathrm{SMSD}}$ is based on difference theory, without the modal parameter of the original structure. FEM numerical simulation to partial crack with different degree is done. The criteria of damage location detection can be obtained by strain mode difference curve through cubic spline interpolation. Also the genetic algorithm box in Matlab is used. It has been possible to identify the damage to a reasonable level of accuracy.
\end{abstract}

\section{Introduction}

Recently the development of new methods of evaluating the integrity of structures has been attached great importance. The vibrational characteristics of structures can be easily alternated once the damage occurs. In order to analyze the state of the structure integrity and the location of structural damage, the modal analysis with vibrational test data is widely employed [1], though the modal vectors were effectively ignored earlier [2,3]. The reason is that the parameters of modal analysis depend only on the mechanical characteristics of 
the structure. Considering this, the characteristics of modal vibration, that is, the natural frequencies, mode shapes, and so on are considered to represent the state of structure [4].

Although damage index based on strain modal shape is much more sensitive [5-8], in most cases, this index can only indicate the damage location both in undamaged and damaged state with modal data. In fact, these kinds of methods are difficult to employ, because the baseline under suddenness and geological disaster is difficult to master. The problem is solved by $\mathrm{Gu}$ et al. [9], who put forward the direct index method of damage location detection $I_{S M S D}$ based on difference theory without baseline modal parameters. The index $I_{\mathrm{SMSD}}$ contains three elements: the effective distance ratio between two adjacent effective extreme points, the absolute difference value of the two effective extremums, and the maximum absolute value of the effective extremum.

Then, the genetic algorithm was gradually applied to solve the problem of damage detection [10]. The method is demonstrated on a simulated beam example and an experimental plate example. Based on the genetic algorithm, Yi and Liu estimated the structural damage with measured dynamic data [11]. Some improved strategies such as multiparent crossover and adjustment of variables are introduced to detect the damage of fixed-end beams and continuous beams. And some satisfied results are achieved. Based on conventional modal analysis theory, Mares and Surace [12] coded a function of binary, and a case of truss-type structure is used to identify the structural damage. And the accurate identification of both the location and the degree of the damage had become possible to be achieved.

Genetic algorithm appears to provide a robust search procedure for solving difficult problems. Due to the way the genetic algorithm explores, the region of interest avoids getting stuck at a particular local minimum and locates the global optimum. To formulate an objective function, with the parameters related to the physical properties and state of the structure, is the aim of the detection of damage in structures. When evaluated with the true parameters, the maximum value of the objective function is obtained. Genetic search algorithm is an optimization procedure which can be employed to determine the values of these parameters by following an iteration process, selecting parameters to maximize the objective function. When the optimization procedure is over, the state of the structure, that is, where and how it is damaged is known.

The objectives of this paper are to develop and apply models to diagnose the damage location and damage degree. This paper is organized as follows: the first section provides a brief introduction to GA, the next section contains results and analyses including performance evaluation of the methodology, and finally the conclusions are presented.

\section{Model Development}

Modal test of continuum structure uses a series displacements of discrete measuring points to describe all order modes of mode functions. The mode results of all order are the ratio of normalization displacements of discrete measuring points. The strain distributing can be calculated by the difference of deformable displacements.

The vibrational differential equation of straight Euler beam in transverse section is

$$
\frac{\partial^{2}}{\partial x^{2}}\left(E I \frac{\partial^{2} u}{\partial x^{2}}\right)+\rho A \frac{\partial^{2} u}{\partial x^{2}}=0
$$


The displacement containing all order modes of displacement $\phi_{r}(x)(r=1,2, \ldots)$ is

$$
u(x, t)=U(x) e^{j \omega t}=\sum_{r=1}^{\infty} \phi_{r}(x) Q_{r} e^{j \omega t}
$$

Curvature mode is the difference of displacement mode.

The discrete curvature mode can be approximately calculated by center difference method as

$$
\phi_{r k}^{\prime \prime}=\frac{\phi_{r(k-1)}-2 \phi_{r k}+\phi_{r(k+1)}}{d x^{2}} \approx \frac{\phi_{r(k-1)}-2 \phi_{r k}+\phi_{r(k+1)}}{h^{2}}
$$

$\phi_{r k}$ is the $r$ th order of displacement amplitude; $k$ is the calculation point; $h$ is the distance between two adjacent calculation points.

Suppose $z(x)$ is the distance from the middle bending surface to any point, then the strain of this point in $x$ direction is

$$
\varepsilon_{z, x}=\frac{\partial u}{\partial x} \approx \frac{\partial^{2} \phi_{r k}}{\partial x^{2}} z(x)=\phi_{r k}^{\prime \prime} z(x)
$$

The relationship between strain mode and curvature mode can be transmitted through formula (2.4). Strain mode shape is more sensitive than displacement mode shape at damage detection.

The curve of strain mode shape has extremum and sharp variation at the damage location for simply supported beam, which is the common structural style. The difference curve of strain mode shape must be smoothed by cubic spline interpolation. Central difference method is a numerical method. The advantages of this method are symmetry, a certain accuracy, and easy to use. The format at the nonboundary nodes are central difference. The format at boundary nodes is eccentric difference.

The beam is separated into $n-1$ units, namely, $n$ nodes. The equidistance is $h$. $\Phi_{r}^{\varepsilon}(x)$ is the $r$ th order strain mode function. The basic equidistance difference format is

$$
\left(\frac{d \Phi_{r}^{\varepsilon}}{d x}\right)_{i}=\frac{\Phi_{r}^{\varepsilon}\left(x_{i+1}\right)-\Phi_{r}^{\varepsilon}\left(x_{i-1}\right)}{2 h}
$$

The difference formulation at boundary node 1 is

$$
\left(\frac{d \Phi_{r}^{\varepsilon}}{d x}\right)_{1}=\frac{\Phi_{r}^{\varepsilon}\left(x_{2}\right)-\Phi_{r}^{\varepsilon}\left(x_{1}\right)}{h}
$$

The difference formulation at boundary node $n$ is

$$
\left(\frac{d \Phi_{r}^{\varepsilon}}{d x}\right)_{n}=\frac{\Phi_{r}^{\varepsilon}\left(x_{n}\right)-\Phi_{r}^{\varepsilon}\left(x_{n-1}\right)}{h} .
$$


When difference curve fits, the 2nd order difference values of two extreme points are

$$
\begin{gathered}
\left(\frac{d^{2} \Phi_{r}^{\varepsilon}}{d x^{2}}\right)_{1}=\frac{\Phi_{r}^{\varepsilon}\left(x_{1}\right)+\Phi_{r}^{\varepsilon}\left(x_{3}\right)-2 \Phi_{r}^{\varepsilon}\left(x_{2}\right)}{2 h^{2}} \\
\left(\frac{d^{2} \Phi_{r}^{\varepsilon}}{d x^{2}}\right)_{n}=\frac{\Phi_{r}^{\varepsilon}\left(x_{n-2}\right)+\Phi_{r}^{\varepsilon}\left(x_{n}\right)-2 \Phi_{r}^{\varepsilon}\left(x_{n-1}\right)}{2 h^{2}} .
\end{gathered}
$$

Nearby the sharp variation section or the peak value of the mode shape, the extreme points at strain mode curve in damage are located. The damage location is at the curve's sharp variation section.

For actual engineering, Extreme point is unnecessarily just zero at the difference of ideal curve. Three reasons are explained as follows: firstly, the space principles of measuring point or unit classification in numerical simulation are not just at the damage location. Secondly, the accuracy problem of both the strain mode test and the computation is to be considered. Thirdly, the accuracy of difference computation is to be considered.

Interpolation method is used to solve the question of the zero point of strain mode difference curve and confirm the extremums. The computational value at nodes is equal to the supposed function. Many function values are calculated and the function curves draw. Cubic spline interpolation is used in this paper, a widely used interpolation method.

\section{Genetic Algorithm}

Complex adaptive systems are extremely difficult to comprehend, when unexpected and unpredictable results occur. Holland succeeds to solve this problem by presenting genetic algorithm. In recent years, genetic algorithms are very popular procedure of robust research for solving maximizing or minimizing a given objective function often subject to some constraints [13-20].

Genetic algorithm derives from the process of natural selection and evolution. Because of the inherent advantage of being able to process with a large population of designs and facilitating arrival at the globally optimal solution, the philosophy of "survival of the fittest" has been adopted. It is necessary to devise a general coding system for the representation of the design variables, namely, a directly analogy of the DNA structure of chromosomes. Most commonly, the design variables are coded by a bit-string which is a binary representation. It can only be coded as integers. The progress of genetic algorithm is in the same way of the natural evolution of a species: the fundamental concepts of reproduction, chromosomal crossover, and occasional mutation of genes. Fitness function, which determines possible solutions to the problem, is used to estimate the quality of the represented solution (chromosome). Crossover is a reproduction operation in GA, which is used to vary the programming from one generation to the next by exchanging genetic information between parent chromosomes.

In applying the evolution theories to designing optimization, a number of candidate design variables either randomly or heuristically are created. Then, they evolve over generations to produce new designs which are "fitter." The "fitness" of the designs is 
evaluated according to the objective function, a specific optimization problem. The point with no further improvement is the solution. This paper applies genetic algorithm to the problem of damage detection using strain mode.

In the application to the damage detection in structures, the aim is to formulate an objective function in terms of damage degree of the structure. The objective function must be formulated in such a way that the maximum value is obtained when evaluated with the true parameters. Genetic algorithm can be employed to determine the values of these parameters by following an iteration process, selecting parameters to maximize the objective function. When the optimization procedure arrives at the solution, the values of the parameters indicate the state of the structure, that is, if, where and how it is damaged. The detailed steps are shown as follows.

Step 1. Initialize: the initial population is generated with binary coding, and each individual represents a initial solution.

Step 2. Individual evaluation: calculate the fitness value of each individual.

Step 3. Convergence judgment: implement Step 4, if the fitness value is still not convergent or cannot reach the limit of iteration times, otherwise, terminate the calculation.

Step 4. Individual crossover, mutation, and selection: return to Step 2 after executing crossover (one-point crossover), mutation (discrete mutation), and selection (sampling randomly) process.

Thus, the search process of genetic search can be seen in Figure 1.

\section{Example}

In the genetic search procedure, selection, crossover, and mutation operators, each with a given probability, are applied to each current population to create the new generations. The results published by DeJong, Gerefenstette, and Schaffer indicate the most appropriate parameter setting: a population size of 20-30 individuals, a crossover rate of $0.6-0.95$, and a mutation rate 0.01-0.02.

Take the "damage rate $6.25 \%$ " as an example to apply the calculation. Figure 2 shows that during the 100 evolutional generations, the result began to converge after the 80th iteration. Therefore, the final result is obtained: in the first order, the best value of $\mathbf{I}_{\mathbf{S M S D}}(\mathbf{2})$ is convergent to 0.0048 .

The mathematical model has solved the maximum of formula (4.5), in which $x \in$ $[1,41]$. Selecting binary code, the swarm number is 10 . The length of binary code is 19 . Crossover probability is 0.95 . The mutation probability is 0.01 . After the 100 times of genetic iterative, the results were close to the data of Table 1.

The example was a numerically simulated simply beams (see Figure 3) with a finite element model. The characteristics of the beam were as follows: length $l=0.4 \mathrm{~m}$, crosssectional area $A=0.0002 \mathrm{~m}^{2}$, in quarter span damage, a one fifth length of the beam width, modulus of elasticity $E=211 \mathrm{Gpa}$, density $\rho=7850 \mathrm{~kg} / \mathrm{m}^{3}$, Poisson radio $\mu=0.33$, damage extent $\alpha_{1}=6.25 \% h, \alpha_{2}=12.5 \% h, \alpha_{3}=25 \% h, \alpha_{4}=31.25 \%$, eight node solid 45 unit in Ansys, 25 grids at transverse section, 16 grids at vertical section, damage width $w=0.002 \mathrm{~m}$.

On the basis of numerical simulation results of the strain modes, the smooth strain mode difference curve can be draw based on central difference and cubic spline interpolation. 


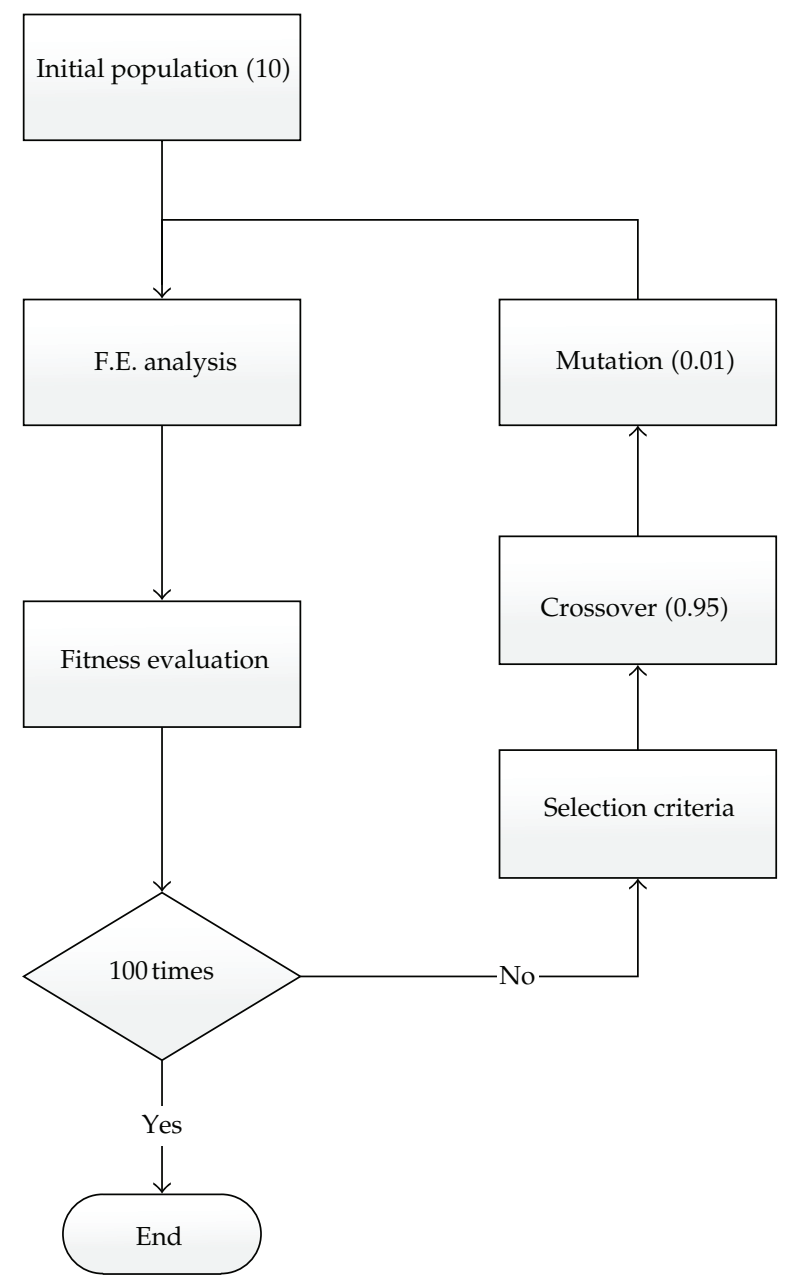

Figure 1: Flowchart of the genetic search.

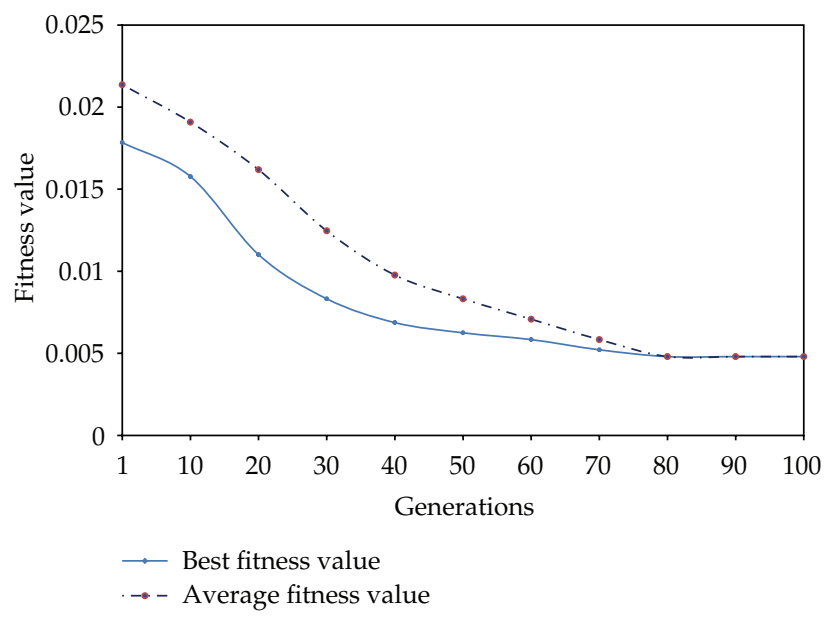

Figure 2: Calculation results. 
Table 1: Damage location indices.

\begin{tabular}{|c|c|c|c|c|c|}
\hline \multirow{2}{*}{ Damage location } & \multirow{2}{*}{ Order } & \multicolumn{4}{|c|}{ Damage degree } \\
\hline & & $6.25 \%$ & $12.5 \%$ & $25 \%$ & $31.25 \%$ \\
\hline \multirow{19}{*}{ Quarter span } & First order & & & & \\
\hline & $I_{\mathrm{SMSD}}(1)$ & 0.0018 & 0.0049 & 0.0111 & 0.0159 \\
\hline & $\mathrm{I}_{\mathrm{SMSD}}(2)$ & 0.0048 & 0.0092 & 0.0197 & 0.0238 \\
\hline & $I_{\mathrm{SMSD}}(3)$ & 0.0011 & 0.0013 & 0.0114 & 0.0167 \\
\hline & $I_{\mathrm{SMSD}}(4)$ & & & 0.0016 & 0.0022 \\
\hline & $I_{\mathrm{SMSD}}(5)$ & & & 0.0010 & \\
\hline & Second order & & & & \\
\hline & $I_{\mathrm{SMSD}}(1)$ & 0.0162 & 0.0205 & 0.0673 & 0.1003 \\
\hline & $\mathrm{I}_{\mathrm{SMSD}}(2)$ & 0.0202 & 0.0497 & 0.1054 & 0.1647 \\
\hline & $I_{\mathrm{SMSD}}(3)$ & 0.0137 & 0.0162 & 0.0655 & 0.0997 \\
\hline & $I_{\mathrm{SMSD}}(4)$ & & 0.0127 & 0.0000078 & 0.0035 \\
\hline & $I_{\mathrm{SMSD}}(5)$ & & & 0.0122 & 0.0105 \\
\hline & $I_{\mathrm{SMSD}}(6)$ & & & & 0.0154 \\
\hline & Third order & & & & \\
\hline & $I_{\mathrm{SMSD}}(1)$ & 0.0787 & 0.0877 & 0.1005 & 0.1335 \\
\hline & $I_{\mathrm{SMSD}}(2)$ & 0.0110 & 0.0661 & 0.1699 & 0.2660 \\
\hline & $I_{\mathrm{SMSD}}(3)$ & 0.0457 & 0.0754 & 0.0885 & 0.1821 \\
\hline & $I_{\mathrm{SMSD}}(4)$ & 0.0544 & 0.0539 & 0.0649 & 0.0644 \\
\hline & $I_{\mathrm{SMSD}}(5)$ & & 0.0701 & 0.0707 & 0.0753 \\
\hline
\end{tabular}

Note: overstriking index and overstriking data correspond to damage location.

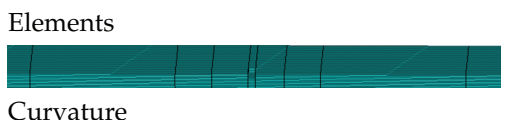

Curvature

Figure 3: FEM modal of local damage in simply supported beam.

The figures (from Figures 4, 5, 6, and 7) were the smooth strain mode difference curves after cubic spline interpolation. The damage extents were $6.25 \%, 12.5 \%, 25 \%$, and $31.25 \%$ of the height.

With the exception of supports, strain mode difference curves have sharp variation at the damage location. For different damage degrees, strain mode curve is slightly different, but the rule has some consistency.

\subsection{Mathematical Model of Direct Index Method of Damage Location}

\subsubsection{Direct Index Method of Damage Location}

From the data change at sharp variation zone between two adjacent extremums, damage index was presented. The extents of the curve's variation were reflected. 


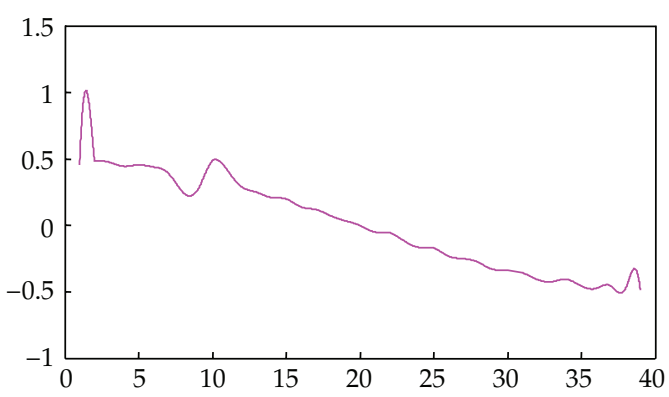

(a) First order

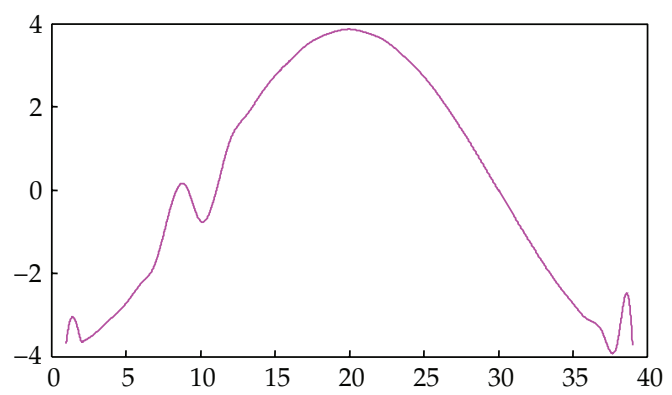

(b) Second order

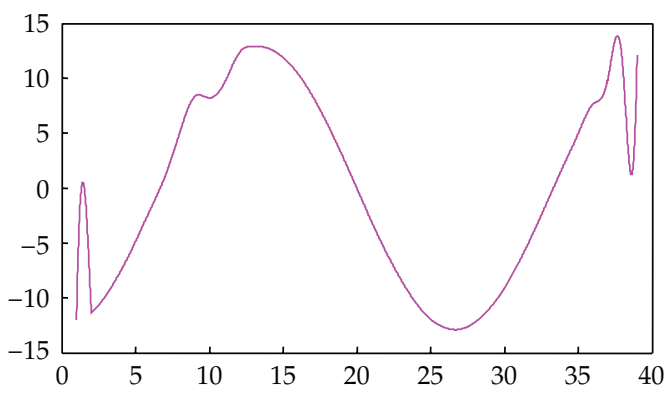

(c) Third order

Figure 4: When damage $6.25 \%$, the first three strain modal difference curves.

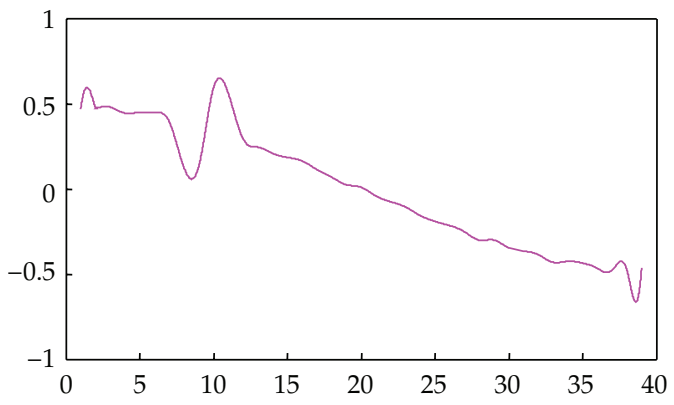

(a) First order

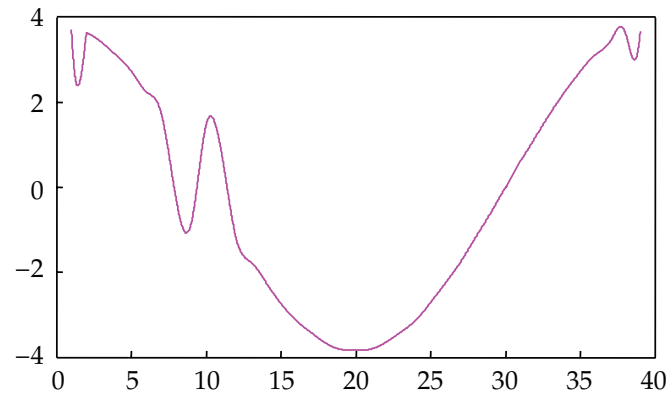

(b) Second order

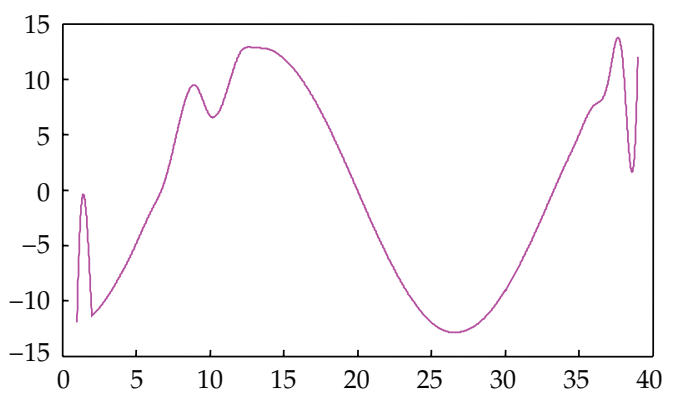

(c) Third order

Figure 5: When damage $12.5 \%$, the first three strain modal difference curves. 


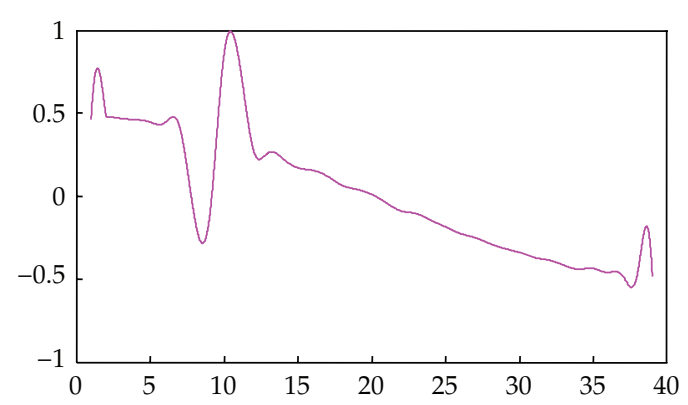

(a) First order

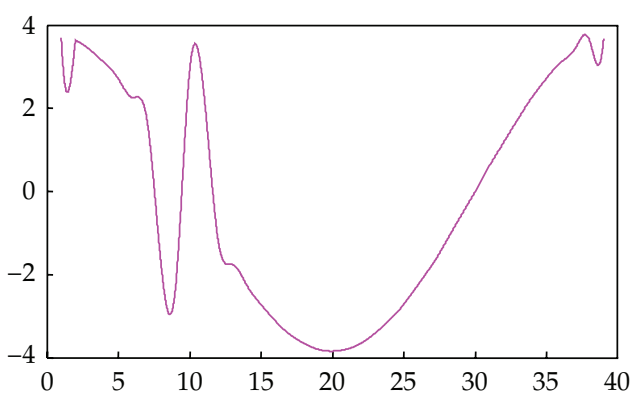

(b) Second order

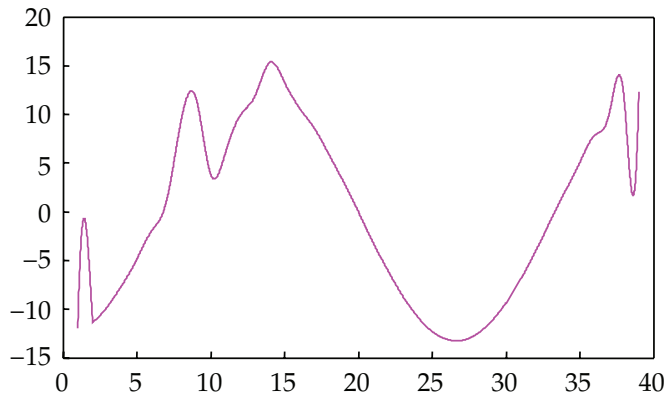

(c) Third order

Figure 6: When damage 25\%, the first three strain modal difference curves.

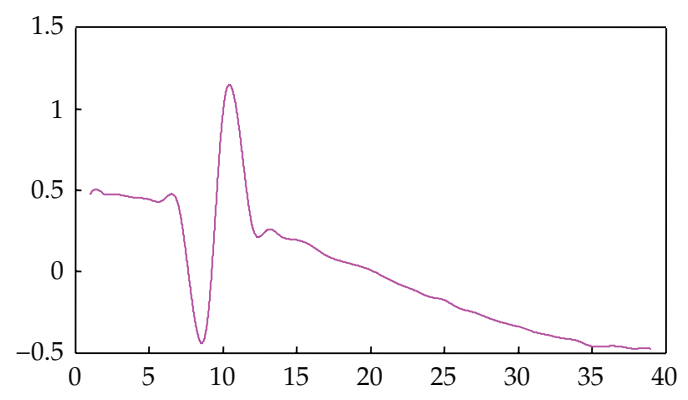

(a) First order

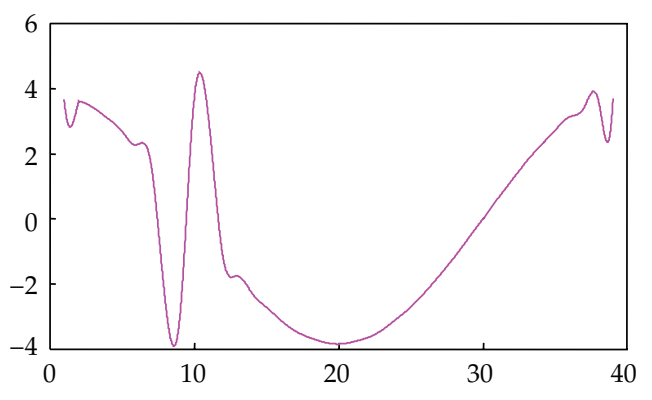

(b) Second order

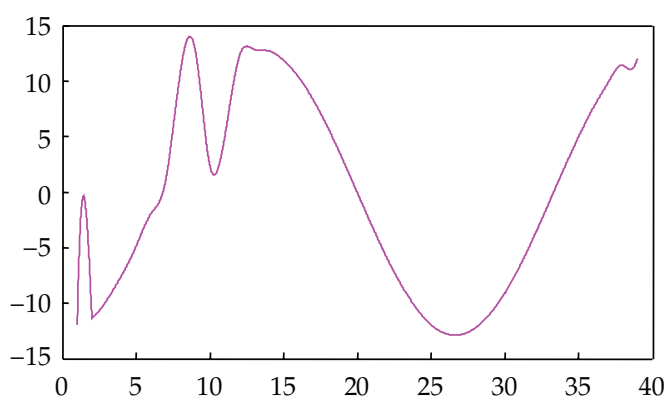

(c) Third order

Figure 7: When damage $31.25 \%$, the first three strain modal difference curves. 


\subsubsection{Effective Distance Ratio}

The distance between two adjacent effective extremums is effective distance. Effective distance ratio is the ratio of effective distance to span.

Suppose: span 1, effective extremum points: $x_{0}, x_{1}, x_{2}, \ldots, x_{p}$, effective extremums: $y_{0}, y_{1}, y_{2}, \ldots, y_{p}$, then the effective distance ratios are

$$
I_{1 j}=\frac{\left(x_{j}-x_{j-1}\right)}{l}, j=(1,2, \ldots, p) .
$$

The smaller distance ratio of the two adjacent effective extremum is, the greater probability of damage is as follows:

$$
I_{2 j}=\left|y_{j 2}-y_{j 1}\right|, \quad j=(1,2, \ldots, p) .
$$

Let

$$
I_{3 j}=\frac{I_{2 j}}{I_{1 j}}, \quad j=(1,2, \ldots, p) .
$$

\subsection{The Absolute Maximum of Effective Extremum: $I_{\mathrm{S} M S \mathrm{D}}$}

As strain mode difference curve of different orders was nonnormalized, the computational results had no comparison.

In order to solve this problem, the results of formula (4.3), divided the absolute maximum of effective extremums of all orders, the data of different orders can be compared

$$
I_{\mathrm{SMSD}}(j)=\frac{I_{2 j}}{I_{1 j} \max \left(\left|y_{0}\right|,\left|y_{1}\right|,\left|y_{2}\right|, \ldots,\left|y_{q}\right|\right)}
$$

Namely,

$$
I_{\mathrm{SMSD}}(j)=l \frac{\left|y_{j 2}-y_{j 1}\right|}{\left(x_{j}-x_{j-1}\right) \max \left(\left|y_{0}\right|,\left|y_{1}\right|,\left|y_{2}\right|, \ldots,\left|y_{q}\right|\right)}
$$

Formula (4.5) is called the direct damage location index. The greater value it is, the more probability of damage occurs. Combined with the rules of difference curve, the damage location can be determined.

Based on formula (4.5), the numerical computation results of damage location indices are listed in Table 1.

From the illustrative examples used, the method using genetic algorithms is very useful to identify both the damage location and the damage degree with a reasonable accuracy. The striking feature of genetic algorithm is that the optimal solution can be determined even with perturbed data having noise. As a parallel search technique, the genetic algorithm makes this approach attractive for problems of large dimensions. 


\section{Conclusion}

The damage indices of the first and second orders are obviously greater than others in the table, and the damage degrees are in accordance with it. The direct index method can accurately detect the damage location and damage degree, especially lower damage. Genetic algorithm is a powerful tool. From the example, accurate identification of both damage location and damage degree had been possible in the structure model. For a protracting beam, there is no macrocrack. The difference curve of strain modes at the damage location has sharp variation, but the numerical value probably is not zero.

\section{Acknowledgment}

This work was supported by National Natural Science Foundation of China (51208079).

\section{References}

[1] M. Basseville, A. Benveniste, B. Gach-Devauchelle et al., "In situ damage monitoring in vibration mechanics: diagnostics and predictive maintenance," Mechanical Systems and Signal Processing, vol. 7, no. 5, pp. 401-423, 1993.

[2] J. K. Vandiver, "Detection of the Structural failure of fixed platforms by measurement of dynamic response," in Proceeding of the Offshore Conference Society of Petroleum Engineering, pp. 243-252, Richaedson, Tex, USA, 1975.

[3] R. N. Coppolino and S. Rubin, "Detectability of structural failure in off-shore platforms by ambient vibration monitoring," in Proceedings of the Offshore Conference Society of Petroleum Engineering, pp. 101-110, Richardson, Tex, USA, May 1975.

[4] D. J. Ewins, 1984 Modal Testing: Theory and Practice, Wiley, New York, 1980.

[5] B. S. Wang, X. B. Liang, Y. Q. Ni et al., "Comparative study of damage indices in application to a long-span suspension bridge," in Advance in Structural Dynamics, pp. 1085-1092, Elsevier Science Ltd, Oxford, UK, 2000.

[6] Y. Y. Lee and K. W. Liew, "Detection of damage location in a beam using the wavelet analysis," International Journal of Structural Stability and Dynamics, vol. 1, no. 3, pp. 455-465, 2001.

[7] J. T. Kim, S. H. Jung, Y. K. Lee, and J. W. Yun, "Damage identification in bridge using vibration-based system identification scheme," in Proceedings of the 18th International Modal Analysis Conference (IMAC '00), pp. 1327-1333, San Antonio, Tex, USA, 2000.

[8] J. T. Kim and N. Stubbs, "Model-uncertainty impact and damage-detection accuracy in plate girder," Journal of Structural Engineering, vol. 121, no. 10, pp. 1409-1417, 1995.

[9] P. Y. Gu, M. Deng, and F. S. Wu, Structure Mode Analysis and Damage Diagnosis, Southeast University Press, Missouri, Mo, USA, 2008.

[10] M. I. Friswell, J. E. T. Penny, and S. D. Garvey, "A combined genetic and eigensensitivity algorithm for the location of damage in structures," Computers and Structures, vol. 69, no. 5, pp. 547-556, 1998.

[11] W. J. Yi and X. Liu, "Damage diagnosis of structures by genetic algorithms," Engineering Mechanics, vol. 18 , no. 2, pp. $64-71,2001$.

[12] C. Mares and C. Surace, "An application of genetic algorithms to identify damage in elastic structures," Journal of Sound and Vibration, vol. 195, no. 2, pp. 195-215, 1996.

[13] S. Rajeev and C. S. Krishnamoorthy, "Discrete optimization of structures using genetic algorithms," Journal of Structural Engineering, vol. 118, no. 5, pp. 1233-1250, 1992.

[14] W. M. Jenkins, "Plane frame optimum design environment based on genetic algorithm," Journal of Structural Engineering, vol. 118, no. 11, pp. 3103-3112, 1992.

[15] W. M. Jenkins, "Towards structural optimization via the genetic algorithm," Computers and Structures, vol. 40, no. 5, pp. 1321-1327, 1991.

[16] A. J. Keane, "Passive vibration control via unusual geometries: the application of genetic algorithm optimization to structural design," Journal of Sound and Vibration, vol. 185, no. 3, pp. 441-453, 1995.

[17] B. Yu and Z. Z. Yang, "An ant colony optimization model: the period vehicle routing problem with time windows," Transportation Research E, vol. 47, no. 2, pp. 166-181, 2011. 
[18] B. Yu, Z. Z. Yang, and S. Li, "Real-time partway deadheading strategy based on transit service reliability assessment," Transportation Research A, vol. 46, no. 8, pp. 1265-1279, 2012.

[19] B. Yu, Z. Z. Yang, P. H. Jin, S.-H. Wu, and B. Z. Yao, “Transit route network design using ant colony optimization," Transportation Research C, vol. 22, pp. 58-75, 2012.

[20] B. Yu, Z. Z. Yang, and B. Yao, "An improved ant colony optimization for vehicle routing problem," European Journal of Operational Research, vol. 196, no. 1, pp. 171-176, 2009. 


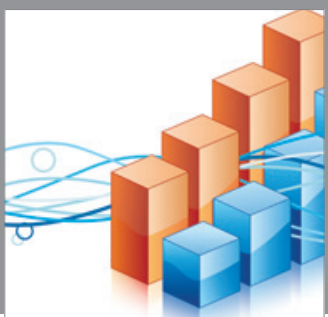

Advances in

Operations Research

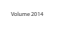

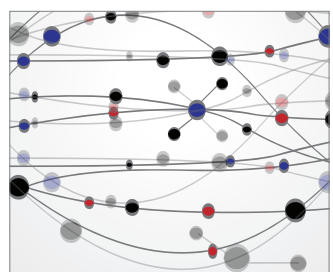

\section{The Scientific} World Journal
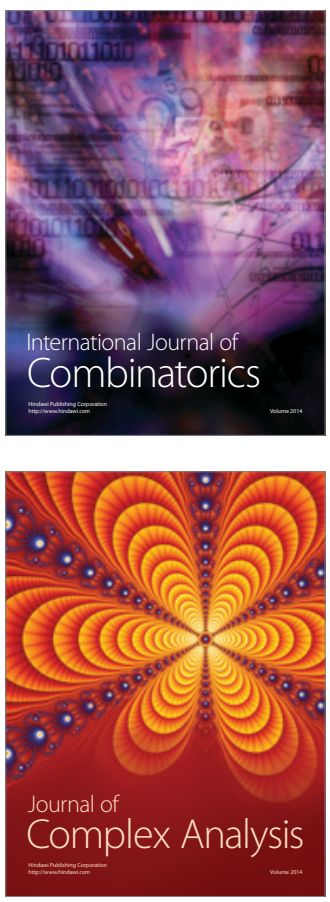

International Journal of

Mathematics and

Mathematical

Sciences
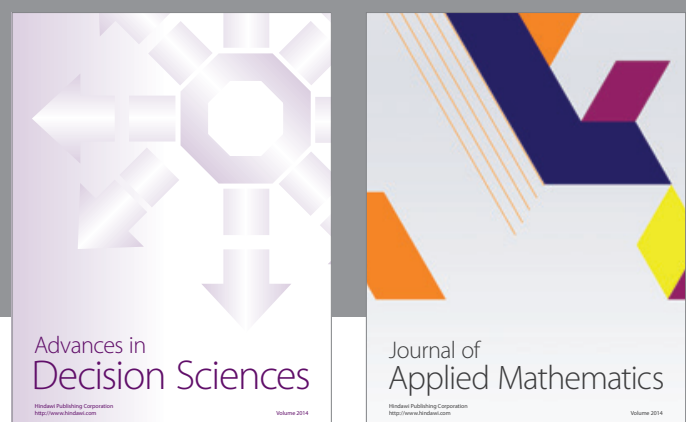

Journal of

Applied Mathematics
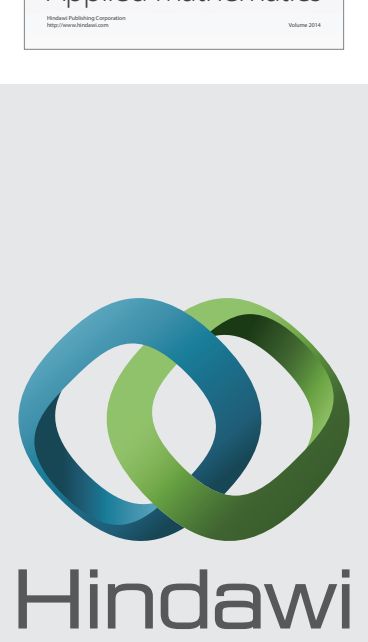

Submit your manuscripts at http://www.hindawi.com
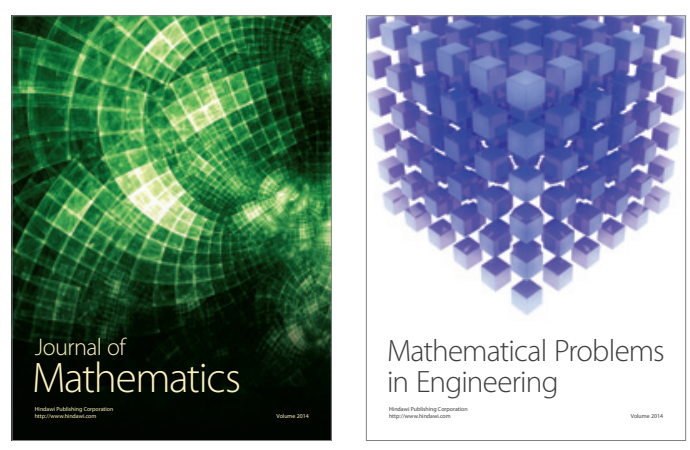

Mathematical Problems in Engineering
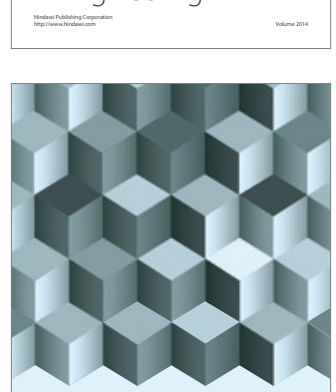

Journal of

Function Spaces
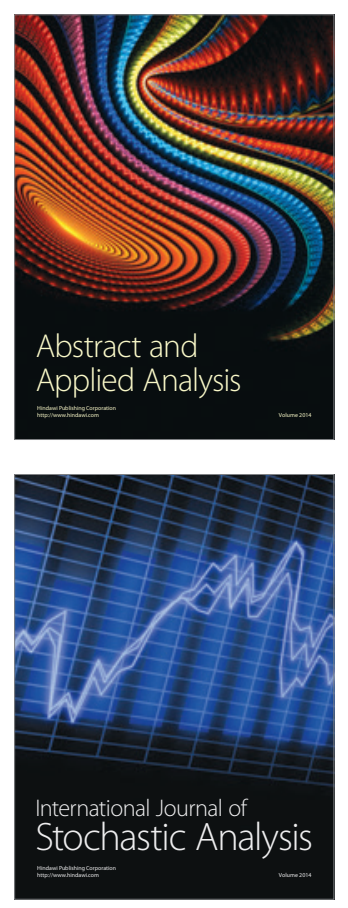

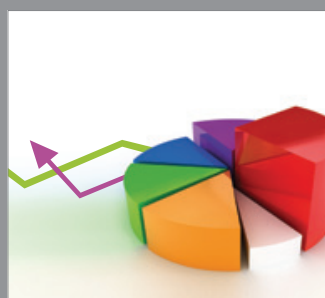

ournal of

Probability and Statistics

Promensencen
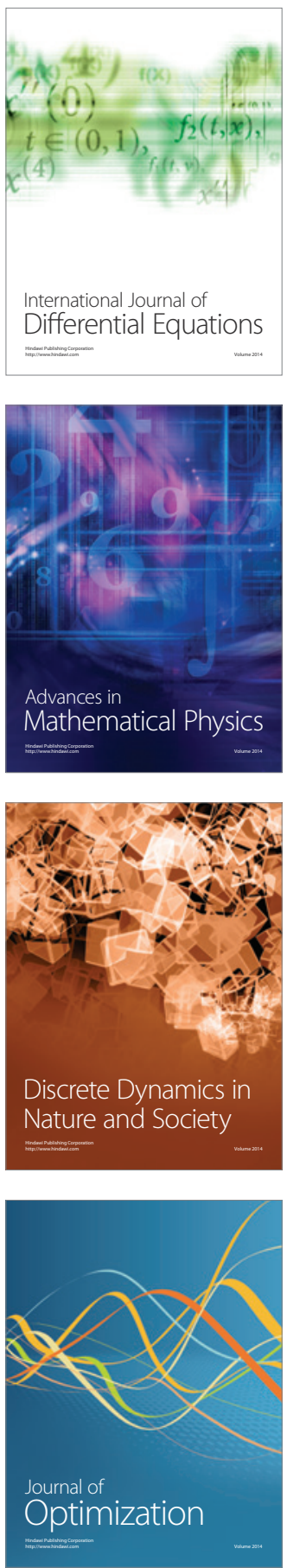